\title{
Características proteanas e afetos sobre carreira de estudantes de Psicologia
}

\author{
Protean career features and affect in the \\ career of Psychology students
}

\author{
Alexsandro Luiz DE ANDRADE \\ Andressa Tonini PISSAIA ${ }^{1}$ \\ Marcelo Zanotti SILVA ${ }^{1}$ \\ Manoela Ziebell de OLIVEIRA ${ }^{2}$
}

\begin{abstract}
Resumo
A presente pesquisa buscou compreender os aspectos proteanos e afetivos relacionados ao projeto de carreira de estudantes universitários do curso de Psicologia, além de explorar como esse projeto pode ser influenciado pelas variáveis comportamento exploratório, personalidade e autoeficácia. O estudo foi realizado com 341 acadêmicos de Psicologia, sendo 156 (45,7\%) de uma faculdade privada e 185 (54,3\%) de uma universidade pública. Para aferir afetos sobre a carreira desenvolveu-se uma escala, a qual apresentou dois fatores: afetos negativos de carreira e afetos positivos de carreira. Ambos apresentaram índices de confiabilidade alfa de Cronbach superiores a 0,80. As variáveis de personalidade neuroticismo, extroversão, conscienciosidade e abertura a ideias, assim como as de autoeficácia e comportamento exploratório, foram preditivas das características da carreira proteana, a saber: autogerenciamento e direcionamento para valores.
\end{abstract}

Palavras-chave: Autoeficácia; Comportamento exploratório; Desenvolvimento de carreira; Personalidade; Psicologia.

\begin{abstract}
This study aimed to understand the features of a protean career and the affective aspects related to the career project of undergraduate Psychology students and to explore how this project can be influenced by exploratory behavior variables, personality, and self-efficacy. The sample was composed of 341 Psychology undergraduate students: 156 (45.7\%) from a private college and $185(54.3 \%)$ from a public university. In order to measure affect in career, a scale composed of two factors was developed: negative affect and positive affect in career. Both factors showed Cronbach's alpha coefficient greater than 0.80 . The personality variables neuroticism, extraversion, conscientiousness, and openness, as well as self-efficacy and exploratory behavior, were shown to be predictive of the protean career dimensions values-driven orientation and self-directedness.
\end{abstract}

Keywords: Self-efficacy; Exploratory behavior; Career development; Personality; Psychology.

vr

1 Universidade Federal do Espírito Santo, Programa de Pós-Graduação em Psicologia, Laboratório de Avaliação e Mensuração Psicológica. Av. Fernando Ferrari, 514, Campus Universitário de Goiabeiras, 29075-910, Vitória, ES, Brasil. Correspondência para/Correspondence to: A.L. DE ANDRADE. E-mail: <alexsandro.deandrade@yahoo.com>.

2 Pontifícia Universidade Católica do Rio Grande do Sul, Departamento de Psicologia, Programa de Pós-Graduação em Psicologia. Porto Alegre, RS, Brasil.

Apoio: Conselho Nacional de Desenvolvimento Científico e Tecnológico. 
No ano de 2012, comemoraram-se os cinquenta anos da regulamentação da profissão de psicólogo no Brasil. Instituída no país a partir da Lei $n^{\circ}$ 4.119, a Psicologia sofreu muitas transformações nas cinco décadas que se seguiram, tanto no que tange à formação quanto às perspectivas profissionais e ao mercado de trabalho (Bedin, Sarriera, \& Paradiso, 2013). Especialmente ao longo da última década, percebeu-se um aumento considerável na quantidade de cursos de graduação e a ampliação das áreas de atuação (Bastos \& Gondim, 2010), o que têm provocado novas exigências e configurações de oportunidades no mercado de trabalho.

A perspectiva de trabalho clínica caracterizou historicamente a profissão e ainda hoje é uma das principais atividades de trabalho para psicólogos habilitados e registrados em conselhos profissionais de classe (Conselho Federal de Psicologia, 2005; Zanelli \& Bastos, 2004). Contudo, as possibilidades de trabalho para o psicólogo vão além da clínica (Furtado, 2012). Os avanços teóricos, as mudanças de mercado, os aspectos políticos e adventos do campo da saúde pública desenharam um novo cenário para a carreira na última década. Nesse contexto, tanto a profissão quanto a sociedade requereram alternativas as quais viabilizaram a inserção profissional de forma segura e duradoura, bem como a extensão do serviço psicológico a grupos minoritários e de baixa renda.

Conforme Yamamoto, Souza, Silva e Zanelli (2010), o número de cursos de graduação em Psicologia no país mais que quadriplicaram nos últimos vinte anos. Observou-se, mais especificamente, uma ligeira e desordenada expansão da graduação em Psicologia a partir da década de 1990 (Lisboa \& Barbosa, 2009). Esse crescimento deve-se, em parte, à expansão do ensino brasileiro e à maior influência do setor privado na formação profissional, responsável, na atualidade, pela formação de $89 \%$ dos psicólogos brasileiros (Yamamoto et al., 2010). Conforme as Diretrizes Curriculares Nacionais para os cursos de graduação em Psicologia do Ministério da Educação (Brasil, 2011), a graduação em Psicologia deve voltar-se para a atuação profissional, pesquisa e ensino, alinhando uma gama de conhecimentos e competências para atuação em diferentes contextos de trabalho.
No que se refere à carreira do psicólogo no cenário atual, verifica-se uma variedade de áreas com diferentes especializações e possibilidades de inserção nas mais diversas instituições, como escolas, hospitais, empresas, consultórios privados, academias, dentre outros (Conselho Federal de Psicologia, 2007; Zanelli \& Bastos, 2004). Ser psicólogo, no Brasil, é atuar em uma profissão a qual oferece muitas possibilidades de inserção profissional, o que facilita alternativas distintas de carreira para os jovens da área (Malvezzi, Souza, \& Zanelli, 2010). Mais especificamente, $70,3 \%$ dos psicólogos plenos e $67,1 \%$ dos recém-formados exercem a profissão e, mesmo em um mercado marcado pela alta competividade e pelos empregos instáveis, dois terços dos psicólogos vivem da profissão, sugerindo que a Psicologia oferece serviços verdadeiramente demandados pela sociedade.

Apesar do crescimento da profissão, a formação no atual contexto de trabalho traz importantes desafios aos futuros psicólogos. Segundo Viriguine, Krawulski, Tavares e Soares (2010), os ambientes de trabalho instáveis, aliados à intensificação da competividade e à flexibilização das relações trabalhistas criam um cenário socioeconômico imprevisível e facilmente mutável. As mudanças que decorrem desse contexto exigem dos profissionais, em diferentes segmentos de atuação, novas habilidades, como a reflexão sobre as diferentes necessidades do mercado e do gerenciamento da própria carreira (Oliveira \& Gomes, 2014). A esse respeito, Gouveia (2011) ressalta que a complexidade do meio acaba por interferir na transição de aluno para trabalhador. Segundo o autor, o emprego estável e a progressão linear trabalhista se tornaram ultrapassados. Como consequência, direções clássicas de carreira são alteradas e, cada vez mais, o indivíduo se torna o grande responsável pelo seu sucesso e crescimento profissional em vez das organizações (Magalhães, 2005). Ao indivíduo cabe, portanto, a responsabilidade pelo gerenciamento e sucesso de sua carreira, o que implica um conjunto de comportamentos estratégicos para o planejamento da mesma.

Nessa conjuntura, o conceito de carreira proteana oferece uma nova perspectiva para o estudo 
das carreiras contemporâneas. Oliveira et al. (2010, p.160) definem carreira proteana como "... um estilo de carreira em que o sucesso, subjetivamente avaliado, é alcançado através do comportamento vocacional autogerenciado e dirigido para valores individuais". Em outras palavras, reflete a atual demanda do mercado de trabalho, o qual propõe o engajamento do indivíduo no sentido de definir e dirigir a própria trajetória.

Para Briscoe, Hall e Frautschy DeMuth (2006), a carreira proteana está predominantemente baseada em dois pilares: direcionamento para valores e autogerenciamento. O primeiro deles se refere aos valores internos que o indivíduo possui e fornecem orientação para a carreira. Esses valores operam como uma bússola interna e dizem respeito às motivações e desejos pessoais os quais orientam o indivíduo ao invés dos fatores externos, como remuneração e oportunidades de ascensão hierárquica (Oliveira et al., 2010). O segundo pilar está relacionado à autogestão da carreira e diz respeito à implicação do indivíduo no processo de obtenção de êxito e realização de seus planos, levando em conta seu desempenho, exigência e aspirações (Magalhães \& Bendassolli, 2013).

Esse novo contexto das carreiras e do mercado traz implicações para a transição universidade-trabalho. O fim do curso universitário é marcado pelo início de uma nova etapa: o exercício da profissão. Porém os recém-formados se deparam com outra realidade: a dificuldade de inserção no mercado, aliada ao sentimento de insegurança e necessidade de intervenção em questões de carreira (Bardagi, Lassance, Paradiso, \& Menezes, 2006). Ao considerar a forma como o mercado de trabalho se organiza na atualidade, é possível perceber que o diploma do curso superior não é mais garantia de trabalho e que há exigências comportamentais e de experiência relevantes (Magalhães \& Bendassolli, 2013). Esse fato, com frequência, elicia diferentes tipos de afeto e sentimentos de medo, incerteza e insegurança entre os jovens profissionais (Teixeira \& Gomes, 2004). Para os recém-formados, é exigida alguma inserção prévia em tarefas mais especializadas e a "habilitação" não é somente configurada pelo diploma, mas também por valores agregados, competências e trajetória profissional (Malvezzi et al., 2010; Teixeira \& Gomes, 2004).

Conforme Pereira (2013), afetos são variáveis inerentes ao processo de carreira. Afeto negativo, por exemplo, no contexto acadêmico, é uma variável associada à perda de foco e à dificuldade de prosseguir em relação aos objetivos (Dietrich, Jokisaari, \& Nurmi, 2012), além de propiciar a perda de desempenho na carreira (Pereira, 2013). Na direção contrária, afeto positivo opera como elemento facilitador de otimismo, satisfação e provável sucesso acadêmico, assim como motivação ocupacional, engajamento e bem-estar no trabalho (Haase, Heckhausen, \& Silbereisen, 2012). Segundo Hutz e Bardagir (2006), as experiências emocionais negativas do tipo depressão e ansiedade são relacionadas a contexto de indecisão de carreira em jovens. Em contrapartida, afetos positivos com a vida estão associados à satisfação com o curso e a carreira em universitários (Bardagi \& Hutz, 2010). Já a visão otimista de futuro caracteriza os universitários concluintes mais motivados diante dos desafios da profissão (Teixeira \& Gomes, 2005).

Como se observou, os aspectos afetivos estão associados a elementos de sucesso e felicidade, bem como de insegurança e ansiedade tanto na carreira quanto na vida pessoal (Dietrich et al., 2012; Haase et al., 2012; Pereira, 2013). Tendo em vista as configurações emergentes da profissão de psicólogo, bem como as transformações das carreiras na contemporaneidade, a dimensão afetiva de carreira será entendida nesta pesquisa como uma variável capaz de influenciar o cumprimento das demandas do processo de formação profissional, bem como o potencial empregatício do estudante de Psicologia. Sendo assim, o presente estudo teve como objetivo compreender alguns elementos afetivos do processo de desenvolvimento de carreira de acadêmicos de Psicologia.

Diante da importância das dimensões afetivas nos diferentes contextos de vida, inclusive profissional, e da não identificação de qualquer medida psicométrica com essa finalidade adaptada ou validada para o contexto brasileiro na revisão prévia à realização deste estudo, propôs-se o desenvolvimento de uma escala de afetos sobre o projeto de 
carreira. Aliado a esse compromisso tecnológico (desenvolvimento de ferramentas), a presente pesquisa também buscou compreender de que forma o processo de desenvolvimento de carreira em estudantes de Psicologia pode ser influenciado por diferentes variáveis, incluindo: os aspectos proteanos de carreira, o comportamento exploratório de mercado, a personalidade e a autoeficácia.

As variáveis investigadas neste estudo dizem respeito a características demandadas pelo mercado dos jovens profissionais. Tendo em vista as mudanças constantes do mercado de trabalho e a tendência à busca de autonomia nas tomadas de decisão sobre a carreira, objetivou-se testar as relações entre os afetos sobre o projeto de carreira e variáveis individuais e comportamentais. Adicionalmente, buscou-se compreender de que forma essas variáveis explicam diferenças no gerenciamento proteano de carreira. Acredita-se que a compreensão dessas relações pode contribuir com o trabalho de orientação profissional e planejamento de carreira de estudantes de Psicologia, especialmente no que tange à transição universidade-mercado de trabalho.

\section{Método}

\section{Participantes}

A amostra do presente estudo foi selecionada utilizando o critério de conveniência (instituições com abertura para realização de pesquisa com estudantes). No entanto, visando à maior extensão das conclusões e ao desenvolvimento de um instrumento passível de uso com diferentes populações em futuros estudos, contou-se com duas instituições de natureza social diferenciada (uma pública e uma privada). No total, 341 estudantes de diferentes períodos de graduação em Psicologia completaram o questionário, sendo que 267 (78,5\%) eram do sexo feminino e $72(21,2 \%)$ do masculino. A média de idade dos participantes foi de 23,4 anos (Desvio-Padrão - $D P=6,8$ anos). Do total de participantes, $156(45,7 \%)$ estavam inscritos em uma faculdade privada e 185 (54,3\%) em uma universidade pública, ambas localizadas na Região Sudeste do territó-

\section{Instrumentos}

O instrumento utilizado foi um questionário autoaplicável com diferentes escalas psicológicas e questões sociodemográficas para caracterização da amostra. As escalas usadas no estudo foram:

a) Escala de Autoeficácia Profissional: instrumento adaptado a partir da versão da Escala de Desenvolvimento Vocacional (Ourique, 2010; Teixeira, 2010). O instrumento adaptado contém 14 itens, os quais avaliaram três dimensões do planejamento de carreira: Autoeficácia profissional, Status financeiro e Realização profissional, todos eles com bons índices de consistência interna;

b) Escala de Gerenciamento Proteano de Carreira para Universitários (EGPC-U) (Borges \& De Andrade, 2014): esta medida contém 18 itens que avaliam as dimensões de autogerenciamento e de direcionamento para valores pessoais, possuindo bons indicadores de validade e precisão;

c) Questionário Exploratório de Mercado: instrumento desenvolvido para este estudo, buscando avaliar o comportamento do indivíduo em relação à busca de oportunidades profissionais, sendo composto por 16 itens e três dimensões: Busca de informação, Busca de emprego e Valores de trabalho (todos os coeficientes são superiores a 0,76 );

d) Marcadores Reduzidos de Personalidade (Hauck Filho, Machado, Teixeira, \& Bandeira, 2012): instrumento composto por 25 adjetivos baseados na Teoria dos Cinco Grandes Fatores de Personalidade (Neuroticismo, Extroversão, Socialização, Conscienciosidade e Abertura), apresentando coeficientes de precisão adequados e maiores que 0,61;

e) Escala de Afetos sobre o Projeto de Carreira (EAPC): instrumento desenvolvido neste estudo com indicadores de precisão superiores a 0,81.

\section{Procedimentos}

\section{Elaboração da Escala de afetos frente ao projeto de carreira}

O instrumento desenvolvido teve como base o Questionário Saúde Geral (Goldberg, 1972) que 
avalia depressão e ansiedade. A partir de fatores de saúde, foi desenvolvida a Escala de Afetos sobre o Projeto de Carreira (EAPC), a qual mensura emoções positivas e negativas que influenciam o processo de planejamento de carreira. $\mathrm{O}$ instrumento foi submetido a um estudo de validação de face dos itens por meio do julgamento de participantes que atendiam aos critérios para a composição da amostra, bem como ao objetivo desta pesquisa. A versão final da EAPC foi composta por 12 itens no formato escala de Likert de 5 pontos ( 1 = "Não me caracteriza" e 5 = "Me caracteriza totalmente"). Todos os itens foram redigidos em formato de afirmativas as quais avaliavam emoções como ansiedade, incerteza, segurança e expectativas sobre afetos relativos ao projeto de carreira.

\section{Coleta de dados}

Inicialmente, o projeto foi enviado para avaliação do Comitê de Ética em Pesquisa com Seres Humanos (CEPSH) por meio do uso da Plataforma Brasil, tendo sido aprovado sob o protocolo de número 248.810. A coleta de dados foi realizada em uma faculdade privada e em uma universidade pública. O procedimento de coleta de dados ocorreu de forma coletiva e voluntária dentro das salas de aula, porém de forma individual e completamente anônima.

\section{Análise de dados}

Os dados da pesquisa foram analisados com auxílio do software Statistical Package for the Social Sciences (SPSS). Primeiramente, realizou-se uma inspeção geral dos questionários a partir de estatísticas exploratórias e descritivas. Para verificar os aspectos de validade e confiabilidade da EAPC, foram realizados procedimentos de análise fatorial e cálculo do coeficiente alfa de Cronbach. Na sequência, foram calculadas as médias para cada dimensão das escalas. Em seguida, procedeu-se as análises de correlação para averiguar as possíveis relações entre as variáveis do estudo. Por fim, foram realizados procedimentos de regressão linear múltipla, tomando como variável dependente as dimensões de carreira proteana (autogerenciamento e direcionamento por valores) e variáveis independentes das demais variáveis psicológicas exploradas neste estudo.

\section{Resultados}

\section{Desenvolvimento da Escala de Afetos sobre o Projeto de Carreira}

Primeiramente, o conjunto de itens da EAPC foi submetido à análise dos componentes principais para verificar a fatoração da matriz de dados. O índice Kaiser-Meyer-Olkin (KMO) obteve o valor de 0,86 e o teste de esfericidade de Bartlett foi significativo $(1908,654$, para $p<0,001)$. Esses valores validam o emprego da técnica de análise fatorial. Para decisão do número de fatores a serem extraídos, optou-se pela informação gerada pelo gráfico scree plot (Enzmann, 1997; Laros \& Puente-Palacios, 2004), o qual sinalizou solução bifatorial ou trifatorial.

Seguiu-se extraindo os fatores a partir dos eixos principais. Optou-se, ao final, pelo modelo com dois fatores, uma vez que este apresentou melhor ajuste à matriz de dados. A rotação escolhida foi a Varimax, pelo fato de ser ortogonal e assegurar que os fatores permaneçam não relacionados (Field, 2009). A disposição dos itens finais da medida é demonstrada na Tabela 1.

A versão final da EAPC contou com 12 itens, dispostos em dois fatores. O primeiro, nomeado de "Afetos Negativos de Carreira", contemplou cinco itens, obtendo coeficiente alfa de Cronbach de 0,89, explicando 37,0\% da variância dos dados. Essa dimensão avaliou emoções negativas frente ao projeto de carreira, envolvendo medo, incerteza e tristeza (e.g., "Tem se sentido deprimido ao pensar no futuro profissional"; "Tem perdido confiança em sua carreira"). O segundo fator, chamado de "Afetos Positivos de Carreira", composto por sete itens, obteve coeficiente alfa de Cronbach de 0,81 e explicou 14,8\% da variância dos dados. Avaliando emoções positivas em relação ao planejamento de carreira, como satisfação profissional (e.g., "A minha 
Tabela 1

Distribuição fatorial dos itens da EAPC - Rotação Varimax

\begin{tabular}{lc}
\hline Itens da EAPC & Fator \\
\cline { 2 - 3 } Tem perdido confiança na sua carreira. & 0,78 \\
Tem se sentido deprimido ao pensar no futuro profissional. & 0,78 \\
Tem se sentido pouco feliz ao pensar sobre o futuro profissional. & 0,76 \\
Tem pensado que não dará certo no futuro profissional. & 0,74 \\
Tem sensação de não superar dificuldades para inserção profissional. & 0,70 \\
A minha vida profissional constrói-se do jeito que eu gostaria que ela fosse. & 0,67 \\
O meu projeto de carreira me parece promissor conforme minhas expectativas de vida. & 0,66 \\
Tem podido concentrar-se no planejamento da sua carreira. & 0,63 \\
Estou satisfeito com a minha vida profissional. & 0,61 \\
Acredita tomar decisões corretas sobre seu futuro profissional. & 0,61 \\
Creio que, ao entrar no mercado, irei conquistar coisas importantes que eu desejo na vida. & 0,54 \\
Se pudesse recomeçar minha formação profissional, não mudaria em quase nada minhas escolhas. & 0,50 \\
\hline Número de itens por fator & 7 \\
Alfa de Cronbach & 5 \\
Porcentagem de variância por fator & 0,89 \\
Média (Desvio-Padrão) & 37,00 \\
\hline
\end{tabular}

Nota: EAPC: Escala de Afetos sobre o Projeto de Carreira; ANC: Afetos Negativos de Carreira; APC: Afetos Positivos de Carreira.

vida profissional constrói-se do jeito que eu gostaria que ela fosse"), segurança (e.g., "Acredita tomar decisões corretas sobre seu futuro profissional"), dentre outros.

\section{Aspectos convergentes e divergentes de afetos sobre o projeto de carreira}

Buscando analisar as relações entre os diferentes construtos deste estudo, bem como levantar os indicadores divergentes e convergentes da EAPC, foram realizadas análises de correlação. As variáveis utilizadas foram: subdimensões de personalidade (Neuroticismo: NEU, Extroversão: EXT, Socialização: SOC, Conscienciosidade: CONSC e Abertura: ABE), afetos de carreira (Afetos Negativos de Carreira: ANC e Afetos Positivos de Carreira: APC), dimensões de carreira proteana (Autogerenciamento: AG e Direcionamento por Valores: DV), autoeficácia (Autoeficácia Financeira: AEF, Autoeficácia de Competência: AEC e Autoeficácia de Realização: AER) e comportamento exploratório (Comportamento Exploratório Informacional: CEl, Comportamento Exploratório de Busca de Emprego: CEBE e Comportamento Exploratório de Valor de Trabalho: (EVT).

682 A Tabela 2 apresenta os resultados.
Os resultados encontrados para as correlações variam de fracos $(r< \pm 0,30, p<0,001)$ a moderados $( \pm 0,30<r< \pm 0,60, p<0,001)$ (Dancey \& Reidy, 2006). Para compreensão dos dados tomou-se como referência as principais correlações das dimensões de APC e ANC.

Com base na dimensão APC, observou-se correlações com: ANC $(r=-0,044)$, AG $(r=0,45)$, AEF $(r=0,30)$, CEI $(r=0,32)$, CEBE $(r=0,42)$ e CEVT $(r=0,44)$. Por sua vez, a dimensão de ANC apresentou correlações significativas de médias a moderadas apenas com a dimensão de NEU $(r=0,35)$, destacando-se também sua relação negativa com AEF $(r=-0,28)$.

Ao analisar as correlações das dimensões proteanas de carreira, verificou-se que Autogerenciamento possui maior correlação com: CEI $(r=0,54)$, CEBE $(r=0,52)$, CEVT $(r=0,45)$ e CONSC $(r=0,34)$. No que tange ao Direcionamento por Valores, verificou-se relações com CEVT $(r=0,35)$ e $\operatorname{ABE}(r=0,21)$.

\section{Preditores de gerenciamento de carreira proteana}

Com o objetivo de explorar modelos preditivos de gerenciamento de carreira proteana em 
Tabela 2

Matriz de correlações

\begin{tabular}{|c|c|c|c|c|c|c|c|c|c|c|c|c|c|c|c|}
\hline & APC & ANC & $A G$ & DV & $\mathrm{AEC}$ & AEF & AER & CEI & CEBE & CEVT & NEU & EXT & SOC & CONSC & $\mathrm{ABE}$ \\
\hline APC & 1 & & & & & & & & & & & & & & \\
\hline ANC & $-0,44^{* *}$ & 1 & & & & & & & & & & & & & \\
\hline$A G$ & $0,45^{* *}$ & $-0,17^{* *}$ & 1 & & & & & & & & & & & & \\
\hline DV & $0,24^{* *}$ & $-0,21^{* *}$ & $0,18^{* *}$ & 1 & & & & & & & & & & & \\
\hline AEC & $0,26^{* *}$ & $-0,12^{*}$ & $0,27^{* *}$ & 0,00 & 1 & & & & & & & & & & \\
\hline AEF & $0,30^{* *}$ & $-0,28^{* *}$ & $0,11^{*}$ & $-0,02$ & $0,17^{* *}$ & 1 & & & & & & & & & \\
\hline AER & 0,10 & $0,14^{*}$ & $0,21^{* *}$ & 0,01 & $0,24^{* *}$ & 0,03 & 1 & & & & & & & & \\
\hline CEI & $0,32^{* *}$ & $-0,12^{*}$ & $0,54^{* *}$ & 0,08 & $0,19^{* *}$ & 0,07 & $0,12^{*}$ & 1 & & & & & & & \\
\hline CEBE & $0,42^{* *}$ & $-0,20^{* *}$ & $0,52^{* *}$ & 0,10 & $0,35^{* *}$ & $0,17^{* *}$ & 0,06 & $0,55^{* *}$ & 1 & & & & & & \\
\hline CEVT & $0,44^{* *}$ & $-0,21^{\star *}$ & $0,45^{* *}$ & $0,35^{* *}$ & $0,14^{*}$ & $0,12^{*}$ & $0,17^{* *}$ & $0,37^{* *}$ & $0,35^{* *}$ & 1 & & & & & \\
\hline NEU & $-0,18^{* *}$ & $0,35^{* *}$ & $-0,05$ & $-0,14^{* *}$ & $-0,10$ & $-0,03$ & $0,16^{* *}$ & $-0,12^{*}$ & $-0,18^{* *}$ & $-0,08$ & 1 & & & & \\
\hline EXT & $0,17^{\star *}$ & $-0,20^{* *}$ & $0,15^{* *}$ & 0,00 & 0,06 & $-0,00$ & 0,04 & $0,15^{* *}$ & 0,10 & 0,10 & $-0,30^{* *}$ & 1 & & & \\
\hline SOC & $0,25^{* *}$ & $-0,11^{*}$ & $0,23^{* *}$ & $0,17^{\star *}$ & $0,11^{*}$ & $0,11^{*}$ & $0,12^{*}$ & $0,16^{* *}$ & $0,11^{*}$ & $0,23^{* *}$ & $-0,10$ & 0,03 & 1 & & \\
\hline CONSC & $0,26^{* *}$ & $-0,20^{* *}$ & $0,34^{* *}$ & 0,08 & 0,10 & $0,13^{*}$ & 0,06 & $0,26^{* *}$ & $0,25^{* *}$ & $0,23^{* *}$ & $-0,11^{*}$ & $-0,02$ & $0,41^{* *}$ & 1 & \\
\hline ABE & $0,27^{* *}$ & $-0,02$ & $0,25^{* *}$ & $0,21^{\star *}$ & $0,13^{*}$ & 0,02 & 0,06 & 0,05 & 0,09 & $0,17^{* *}$ & 0,07 & $0,13^{*}$ & 0,07 & $-0,03$ & 1 \\
\hline
\end{tabular}

Nota: *Valor de $r$ significativo para $p<0,005 ;{ }^{* *}$ Valor de $r$ significativo para $p<0,001$

APC: Afetos Positivos de Carreira; ANC: Afetos Negativos de Carreira; AG: Autogerenciamento; DV: Direcionamento por Valores; AEC: Autoeficácia de Competência; AEF: Autoeficácia Financeira; AER: Autoeficácia de Realização; CEl: Comportamento Exploratório Informacional; CEBE: Comportamento Exploratório de Busca de Emprego; CEVT: Comportamento Exploratório de Valor de Trabalho; NEU: Neuroticismo; EXT: Extroversão; SOC: Socialização; CONSC: Conscienciosidade; ABE: Abertura.

Tabela 3

Modelos de regressões lineares múltiplas das variáveis dependentes de carreira proteana (Autogerenciamento e Direcionamento por Valores)

\begin{tabular}{lcccc}
\hline \multirow{2}{*}{ Preditores } & \multicolumn{2}{c}{ Autogerenciamento $R^{2}=47,40$} & & \multicolumn{2}{c}{ Direcionamento por Valores $R^{2}=18,30$} \\
\cline { 2 - 3 } & $\beta$ & $p$ & 0 & 0,493 \\
\hline APC & 0,12 & 0,021 & 0,05 & 0,014 \\
ANC & 0,00 & 0,956 & $-0,15$ & 0,175 \\
AEC & 0,04 & 0,315 & $-0,08$ & 0,045 \\
AEF & $-0,03$ & 0,525 & $-0,11$ & 0,988 \\
AER & 0,08 & 0,067 & 0,00 & 0,318 \\
CEI & 0,26 & 0,000 & $-0,06$ & 0,975 \\
CEBE & 0,21 & 0,00 & 0,000 \\
CEVT & 0,15 & 0,000 & 0,31 & 0,036 \\
NEU & 0,07 & 0,001 & $-0,12$ & 0,029 \\
EXT & 0,06 & 0,118 & $-0,12$ & 0,058 \\
SOC & 0,02 & 0,193 & $-0,05$ & 0,411 \\
CONSC & 0,16 & 0,636 & 0,17 & 0,001 \\
ABE & 0,14 & 0,001 & 0,001 & \\
\hline
\end{tabular}

Nota: APC: Afetos Positivos sobre a Carreira; ANC: Afetos Negativos sobre a Carreira; AEC: Autoeficácia de Competência; AEF: Autoeficácia Financeira; AER: Autoeficácia de Realização; CEl: Comportamento Exploratório Informacional; CEBE: Comportamento Exploratório de Busca de Emprego; CEVT: Comportamento Exploratório de Valor de Trabalho.

Cinco fatores de personalidade: NEU: Neuroticismo; EXT: Extroversão; SOC: Socialização; CONSC: Conscienciosidade; ABE: Abertura. $R^{2}: R^{2}$ ajustado.

estudantes de Psicologia, foram realizadas duas análises de regressão múltipla. Como variáveis dependentes, foram utilizados os subfatores de carreira proteana (Autogerenciamento e Direcionamento para Valores) e, como variáveis independentes, os afetos de carreira (autoeficácia, compor- tamento exploratório informacional e personalidade). A Tabela 3 apresenta os resultados.

O primeiro modelo preditivo para Autogerenciamento explicou 47,4\% da variância dos dados $\left(F_{13,327}=24,614 ; p<0,001\right)$. As variáveis significativas do modelo foram: de personalidade - CONSC 
$(\beta=0,16, p<0,001)$ e $\operatorname{ABE}(\beta=0,14, p<0,001)$; de afetos de carreira - APC $(\beta=0,12, p<0,02)$; de comportamento exploratório - CEI $(\beta=0,26, p<0,001)$, CEBE $(\beta=0,21, p<0,001)$ e CEVET $(\beta=0,15$, $p<0,001)$. O segundo modelo para Direcionamento de Valores explicou 18,30\% da variância dos dados $\left(F_{13,327}=24,614 ; p<0,001\right)$, sendo significativas as variáveis: personalidade - NEU $(\beta=-0,12, p<0,03)$, EXT $(\beta=-0,12, p<0,02)$ e ABE $(\beta=0,17, p<0,001)$; afetos de carreira - ANC $(\beta=-0,15, p<0,01)$; autoeficácia - AEF $(\beta=-0,11, p<0,04)$; e comportamento exploratório - CEVT $(\beta=0,31, p<0,001)$.

\section{Discussão}

Contemplando um dos objetivos iniciais, pode-se constatar que a EAPC apresentou indicadores de validade de construto e discriminante aceitáveis para seu uso em contextos de pesquisa e avaliação de programas de orientação de carreira. Também apresentou índices satisfatórios de precisão para ambos os fatores extraídos (Nunnally, 1978).

Com base nos dados obtidos através da correlação com outros construtos, pode-se observar que os afetos positivos sobre o projeto de carreira correlacionam-se positivamente com os fatores: autogerenciamento, autoeficácia financeira e fatores do comportamento exploratório (informacional, busca de emprego e valor de trabalho). Estudantes de Psicologia que demonstram maiores níveis de afetos positivos de carreira sentem-se mais confiantes quanto às suas habilidades pessoais (autoeficácia). A autoeficácia profissional diz respeito à crença e à confiança que o indivíduo tem sobre sua capacidade para lidar e executar atividades relacionadas à profissão (Ourique, 2010). Portanto, compreende-se que estudantes os quais se sentem capazes de lidar com as atividades relacionadas à sua formação, acreditando que irão obter resultados positivos ao executá-las, tendem a se comprometer ativamente com o seu futuro profissional. Da mesma maneira, tendem a se engajar na busca de informações acerca do mundo do trabalho (comportamento exploratório), assumindo a responsabilidade pelo próprio sucesso profissional (autogerenciamento).
Teixeira e Gomes (2004) sinalizam que pessoas as quais possuem características elevadas de autogerenciamento comumente colocam-se em posições mais centrais de carreira, sendo mais ativas em comportamentos de busca de emprego, entre outros. Especificamente em pesquisa com amostra de estudantes ingressantes no curso de Psicologia, Pérez (2009) constatou que o otimismo, assim como a presença de indicadores elevados de saúde mental e satisfação geral com a vida são variáveis relacionadas ao não abandono do curso e à maior satisfação com a carreira. Os afetos negativos sobre o projeto de carreira apresentaram relação positiva com a dimensão de personalidade neuroticismo, caracterizada por padrões emocionais instáveis, além da presença de emoções como raiva, ansiedade e depressão (Nunes, Hutz, \& Nunes, 2010; Zanon, Bastianello, Pacico, \& Hutz, 2013). Esses resultados sinalizam que o pessimismo sobre o futuro profissional, aliado a características pessoais de instabilidade emocional, podem afetar a construção de estratégias adequadas de planejamento de carreira.

Quanto ao modelo preditivo para Autogerenciamento $(A G)$, observa-se a importância das características de personalidade de conscienciosidade e abertura. O primeiro é um indicador de responsabilidade, organização e planejamento e o segundo está associado à flexibilidade de ideias e busca por novas experiências (Costa Júnior \& McCrae, 2007; Nunes et al., 2010). Essas características predizem a orientação proteana de carreira, sinalizando que dimensões individuais de gerenciamento de vida também são pertinentes para contextos profissionais (Sullivan \& Baruch, 2009). Outras variáveis também se mostraram preditivas: os afetos positivos de carreira e os três tipos de comportamento exploratório. Desta forma, infere-se que pessoas com autogerenciamento elevado adaptam-se com maior facilidade às demandas externas do mercado, sendo que essa flexibilidade acaba por influenciar de maneira positiva a sua percepção de oportunidades de carreira (Noronha \& Mansão, 2012; Paschoal \& Tamayo, 2008; Teixeira, 2010).

O modelo de Direcionamento para Valores (DV) obteve índices de variância explicada menores que o modelo de Autogerenciamento, evidenciando 
a necessidade de maiores estudos sobre essa dimensão. Resultados semelhantes foram encontrados recentemente por Borges e De Andrade (2014) em uma amostra de universitários, destacando a dificuldade de compreensão dos aspectos de valores de carreira e trabalho dos futuros profissionais. É possível que essa dificuldade deva-se ao fato desses valores estarem atrelados a esquemas cognitivos diferentes para cada indivíduo e que, portanto, guiam seu comportamento e escolhas no papel de trabalhador (Brown, 2002). Assim, a variedade de esquemas cognitivos dos estudantes os quais compuseram a amostra justificaria uma menor variância explicada para o direcionamento de valores.

Todavia, o presente modelo teve como variáveis significativas as facetas de personalidade: neuroticismo (negativamente), extroversão (negativamente) e abertura. Sinalizando que dirigir a carreira por valores não se associa com características emocionais instáveis, tampouco com extroversão elevada. Esse resultado remete à ideia de que valores e crenças relativamente estáveis ao longo do tempo funcionam como padrões, os quais guiam a maneira de viver do indivíduo (Brown, 2002). Adicionalmente, sugere um perfil de maturidade emocional e tendência individualizante de buscar estimulação e companhia de pessoas com níveis semelhantes de DV, além de criatividade e inovação, características do traço de abertura. Outras variáveis preditivas do modelo de DV foram: afetos negativos de carreira (negativamente) e comportamento exploratório de valor de trabalho. Esses indivíduos, quando buscam emprego, são mais sensíveis aos valores envolvidos no trabalho do que a características como salário e progressões disponíveis, apresentando um padrão de busca por ambientes que possam fornecer novas experiências profissionais, demonstrando menor tendência a se influenciar por afetos negativos em relação à carreira (Briscoe et al., 2006; Hall \& Chandler, 2005).

Os resultados deste estudo são relevantes para o planejamento de carreira de estudantes de cursos de Psicologia, bem como estudantes de outras áreas, especialmente quando se considera as mudanças recentes no mercado de trabalho e o fato dos vínculos de emprego estarem se tornando mais instáveis. A responsabilidade envolvida na passagem da universidade para o mercado de trabalho e na busca por uma colocação profissional, demanda que os estudantes estejam atentos às oportunidades oferecidas. Além disso, há uma expectativa de que busquem conhecer as competências específicas de cada área de atuação e identificar as características pessoais capazes de viabilizar a entrada nos diferentes espaços da profissão. Esse processo tende a mobilizar afetos positivos e negativos aos quais estudiosos e práticos da orientação profissional e do aconselhamento de carreira devem estar atentos, uma vez que esses afetos podem favorecer a maneira como o indivíduo vê a si mesmo e as outras pessoas.

Segundo Noronha e Mansão (2012), os afetos negativos estão associados aos eventos da vida compreendidos como ameaçadores, podendo implicar perdas e rompimento de metas, enquanto os positivos tendem a gerar mais prazer para vivenciar situações cotidianas. Portanto, os orientadores profissionais podem assumir um importante papel ao auxiliar os estudantes na realização do processo de autoconhecimento e de identificação dos afetos despertados por esse processo, bem como de características individuais, como a autoeficácia profissional e o comportamento exploratório - fundamentais quando se considera um mercado de trabalho em contínua transformação.

De forma semelhante ao que ocorreu em diversas áreas nas últimas décadas, muitas mudanças se operaram no que concerne à profissão de psicólogo, especialmente no Brasil. Como destaca Furtado (2012), as possibilidades de trabalho para esse profissional têm se expandido para além da clínica particular. Novas áreas ganham força como oportunidades para o exercício da profissão (e.g., trabalho com comunidades e políticas públicas) e como alternativas viáveis às mais tradicionais, enquanto outras são caracterizadas por ampla transformação (e.g., Psicologia Organizacional e do Trabalho).

São reflexos das mudanças ocorridas na área a diversificação dos currículos dos cursos de Psicologia, especialmente em instituições de ensino (universidades e faculdades) privadas, bem como o 
aumento do número de estudantes em formação e de profissionais formados - 216 mil inscritos ativos no Sistema Conselhos de Psicologia no ano de 2012. Esses números evidenciam a necessidade dos estudantes se atualizarem e desenvolverem as competências necessárias à inserção no mercado e à atuação profissional em um contexto de mudanças. Mais especificamente, é preciso que se preparem para postos de trabalho mais fluidos e menos definidos e que demonstrem prontidão para atender às exigências do mercado (Furtado, 2012).

Nesse sentido, profissionais que atuam com orientação e aconselhamento de carreira podem ter uma contribuição extremamente relevante ao viabilizar o entendimento sobre como são planejadas as ações futuras de carreira e poderão assistir os estudantes na execução desse plano. Esse tipo de contribuição pode se dar por meio do desenvolvimento de instrumentos e de evidências de pesquisa os quais contribuam para as práticas de aconselhamento e orientação de carreira, como pretendeu fazer o presente estudo.

\section{Considerações Finais}

A relevância desta pesquisa reside no fato de que a transição universidade-mercado de trabalho se caracteriza por um período de acentuado conflito, no qual o jovem se depara com a responsabilidade de uma decisão. A Psicologia, enquanto profissão, dedica-se a diferentes frentes nesse processo: promove autoconhecimento, auxilia no enfrentamento de conflitos e eventos negativos, promove conhecimento sobre como operam as variáveis de exploração de carreira entre os futuros psicólogos e a criação de subsídios de enfrentamento dos desafios do mercado.

Durante esse processo, intervenções e questões sobre o futuro profissional podem favorecer um ingresso mais eficiente no mundo laboral, de modo que o jovem reconheça suas inquietações, motivações e interesses e desenvolva uma perspectiva de futuro mais clara. Quanto aos achados do estudo, os mesmos são iniciais e restringem-se a uma amostra de jovens de uma localização do

686 Sudeste do Brasil. Novas pesquisas com instru- mentos e participantes diferenciados podem favorecer a melhor compreensão das relações aqui investigadas.

\section{Colaboradores}

A.L. ANDRADE, A.T. PISSAIA e M.Z. SILVA participaram na elaboração de estratégia experimental, coleta de dados, tabulação e discussão dos resultados e elaboração do artigo. M.Z. OLIVEIRA participou na discussão dos resultados e na elaboração e revisão do artigo.

\section{Referências}

Bardagi, M., Lassance, M. C. P., Paradiso, Â. C., \& Menezes, I. A. (2006). Career choice and insertion in labor market: Perceptions from college seniors. Psicologia Escolar e Educacional, 10(1), 69-82. http://dx.doi.org/ 10.1590/S1413-85572006000100007

Bardagi, M. P., \& Hutz, C. S. (2010). Satisfação de vida, comprometimento com a carreira e exploração vocacional em estudantes universitários. Arquivos Brasileiros de Psicologia, 62(1), 159-170. Recuperado em junho 7, 2014, de http://pepsic.bvsalud.org/scielo. php?script=sci_arttext\&pid=S1809-5267201000010 0016\&lng=pt\&nrm=iso

Bastos, A. V. B., \& Gondim, S. M. G. (2010). O trabalho do psicólogo no Brasil. Porto Alegre: Artmed.

Bedin, L. M., Sarriera, J. C., \& Paradiso, Â. C. (2013). Desenvolvimento de carreira em psicólogos: tarefas evolutivas de estabelecimento. Revista Brasileira de Orientação Profissional, 14(1), 87-98. Recuperado em abril 10, 2014, de http://pepsic.bvsalud.org/scielo. php?script=sci_arttext $\&$ pid $=$ S1679-3390201300 0100009\&lng=pt\&nrm=iso\&tlng=pt

Borges, L. F. L., \& De Andrade, A. L. (2014). Gerenciamento proteano de carreira entre universitários (Dissertação de mestrado não-publicada). Universidade Federal do Espírito Santo. Recuperado em junho 11, 2014, de http://repositorio.ufes.br/bitstream/10/1157/ 1/Dissertacao.LudmilaFerreiraLiberato.texto.pdf

Brasil. Ministério da Educação. (2011). Resolução nº 5, de 15 de março de 2011. Institui as Diretrizes Curriculares Nacionais para os cursos de graduação em Psicologia, estabelecendo normas para o projeto pedagógico complementar para a Formação de Professores de Psicologia. Diário Oficial da União, n 51, março 2011, Seção 1, p.19-20.

Briscoe, J. P., Hall, D. T., \& Frautschy DeMuth, R. L. (2006). Protean and boundaryless careers: An empirical exploration. Journal of Vocational Behavior, 69(1), 30-47. http://dx.doi.org/10.1016/j.jvb.2005.09.003 
Brown, D. (2002). Introduction to theories of career development and choice: Origins, evolution, and current efforts. In D. Brown (Ed.), Career choice and development (4 ${ }^{\text {th }}$ ed., pp.3-23). San Francisco: John Wiley \& Sons.

Conselho Federal de Psicologia. (2005). Código de ética profissional do psicólogo. Brasília: Autor.

Conselho Federal de Psicologia. (2007). Resolução CFP n 013/2007. Institui a Consolidação das Resoluções relativas ao Título Profissional de Especialista em Psicologia e dispõe sobre normas e procedimentos para seu registro. Brasília: Autor. Recuperado em março 4, 2014, de http://site.cfp.org.br/wp-content/ uploads/2007/09/resolucao2007_13.pdf

Costa Júnior, P. T., \& McCrae, R. R. (2007). NEO PI-R: inventário de personalidade $N E O$ revisado e inventário de cinco fatores NEO revisado NEO-FFI-R (versão curta). São Paulo: Vetor.

Dancey, C. P., \& Reidy, J. (2006). Estatística sem matemática para Psicologia: usando SPSS para Windows. Porto Alegre: Artmed.

Dietrich, J., Jokisaari, M., \& Nurmi, J. E. (2012). Workrelated goal appraisals and stress during the transition from education to work. Journal of Vocational Behavior, 80(1), 82-92. http://dx.doi.org/10.1016/j.jvb. 2011.07.004

Enzmann, D. (1997). RanEigen: A program to determine the parallel analysis criterion for the number of principal components. Applied Psychological Measurement, 21(3), 232-232. http://dx.doi.org/ 10.1177/01466216970213003

Field, A. (2009). Descobrindo a estatística usando o SPSS ( $2^{a}$ ed.). Porto Alegre: Artmed.

Furtado, O. (2012). Cinquenta anos de Psicologia no Brasil: a construção social de uma profissão. Psicologia: Ciência e Profissão, 32(Esp.), 66-85. http://dx.doi.org/ 10.1590/S1414-98932012000500006

Goldberg, D. P. (1972). The detection of psychiatric illness by questionnaire: A technique for the identification and assessment of non-psychotic psychiatric illness. London: Oxford University Press.

Gouveia, B. M. (2011). Empregabilidade e auto-eficácia na transição para o trabalho em alunos finalistas de cursos profissionais (Dissertação de mestrado não-publicada). Universidade de Lisboa, Portugal. Recuperado em março 20, 2014, de http://repositorio.ul.pt/ handle/10451/5077

Haase, C. M., Heckhausen, J., \& Silbereisen, R. K. (2012). The interplay of occupational motivation and wellbeing during the transition from university to work. Developmental Psychology, 48(6), 1739-1751. http:// dx.doi.org/10.1037/a0026641

Hall, D. T., \& Chandler, D. E. (2005). Psychological success: When the career is acalling. Journal of Organizational Behavior, 26(2), 155-176. http://dx.doi.org/10.1002/ job.301
Hauck Filho, N., Machado, W. L., Teixeira, M. A. P., \& Bandeira, D. R. (2012). Evidências de validade de marcadores reduzidos para a avaliação da personalidade no modelo dos cinco grandes fatores. Psicologia: Teoria e Pesquisa, 28(4), 417-423. Recuperado em março 20, 2014, de http://www.scielo.br/pdf/ptp/v28n4/ 07.pdf

Hutz, C. S., \& Bardagir, M. P. (2006). Indecisão profissional, ansiedade e depressão na adolescência: a influência dos estilos parentais. Psico-USF, 11(1), 65-73. http:// dx.doi.org/10.1590/S1413-82712006000100008

Laros, J. A., \& Puente-Palacios, K. E. (2004). Validação cruzada de uma escala de clima organizacional. Estudos de Psicologia (Natal), 9(1), 113-119. http://dx.doi. org/10.1590/S1413-294X2004000100013

Lisboa, F. S., \& Barbosa, A. J. G. (2009). Psychology education in Brazil: A profile of undergraduation programs. Psicologia: Ciência e Profissão, 29(4), 718-737. http://dx.doi.org/10.1590/\$1414-98932009 000400006

Magalhães, M. O. (2005). Personalidades vocacionais e desenvolvimento na vida adulta: generatividade e carreira profissional (Tese de doutorado não-publicada). Universidade Federal do Rio Grande do Sul, Porto Alegre. Recuperado em abril 26, 2014, de http://www. lume.ufrgs.br/handle/10183/4934

Magalhães, M. O., \& Bendassolli, P. F. (2013). Desenvolvimento de carreira nas organizações. In L. O. Borges \& L. Mourão (Orgs.), O trabalho e as organizações: modos de atuação a partir da Psicologia (pp.433-464). Porto Alegre: Artmed.

Malvezzi, S., Souza, J. A. J., \& Zanelli, J. C. (2010). Inserção no mercado de trabalho: os psicólogos recém-formados. In S. M. G. Gondim \& A. V. B. Bastos (Orgs.), O trabalho do psicólogo no Brasil. Porto Alegre: Artmed.

Noronha, A. P. P., \& Mansão, C. S. M. (2012). Interesses profissionais e afetos positivos e negativos: estudo exploratório com estudantes de ensino médio. PsicoUSF, 17(2), 323-331. http://dx.doi.org/10.1590/S1 413-82712012000200016

Nunes, C., Hutz, C. S., \& Nunes, M. F. O. (2010). Bateria Fatorial de Personalidade (BFP): manual técnico. São Paulo: Casa do Psicólogo.

Nunnally, J. C. (1978). Psychomtietric theory $\left(2^{\text {nd }}\right.$ ed. pp.701). New York: McGraw-Hill.

Oliveira, M. Z., \& Gomes, W. B. (2014). Estilos reflexivos e atitudes de carreira proteana e sem fronteiras nas organizações contemporâneas brasileiras. Revista Psicologia, 14(1), 105-118. Recuperado em junho 11, 2014, de http://pepsic.bvsalud.org/scielo.php?script= sci_arttext\&pid=S1984-66572014000100009\&lng= pt\&nrm=iso\&tlng=pt

Oliveira, M. Z., Zanon, C., Silva, I. S., Pinhatti, M. M., Gomes, W. B., \& Gauer, G. (2010). Avaliação do autoge- 
renciamento e do direcionamento de carreira: estrutura fatorial da escala de atitudes de carreira proteana. Gerais: Revista Interinstitucional de Psicologia, 2(2), 160-169. Recuperado em março 3, 2014, de http:// www.fafich.ufmg.br/gerais/index.php/gerais/article/ view/99/57

Ourique, L. R. (2010). Auto-eficácia e personalidade no planejamento de carreira de universitários (Dissertação de mestrado não-publicada). Universidade Federal do Rio Grande do Sul, Porto Alegre. Recuperado em março 10, 2014, de http://www.lume.ufrgs.br/handle/ $10183 / 27840$

Paschoal, T., \& Tamayo, A. (2008). Construction and validation of the work well-being scale. Avaliação Psicológica, 7(1), 11-22. Recuperado em abril 15, 2014, de http://pepsic.bvsalud.org/scielo.php?script=sci_ arttext\&pid=S1677-04712008000100004

Pereira, D. F. (2013). Planejamento de carreira: estudo exploratório na modalidade a distância (Tese de doutorado não-publicada). Universidade Federal do Rio Grande do Sul, Porto Alegre. Recuperado em junho 11, 2014, de http://www.lume.ufrgs.br/handle/10183/ 87981

Pérez, L. C. (2009). Optimism and positive health as predictors of adjustment to university life. Acta Colombiana de Psicología, 12(1), 95-107. Recuperado em junho 11, 2014, de http://www.scielo.org.co/ scielo.php?script=sci_arttext\&pid=S0123-9155200 9000100009\&lng=en\&nrm=iso\&tlng=es

Sullivan, S. E., \& Baruch, Y. (2009). Advances in career theory and research: A critical review and agenda for future exploration. Journal of Management, 35(6), 1542-1571. http://dx.doi.org/10.1177/0149206309 350082

Teixeira, M. A. P. (2010). Escalas de desenvolvimento vocacional: relatório preliminar (Manuscrito não-publicado). Universidade Federal do Rio Grande do Sul, Porto Alegre.
Teixeira, M. A. P., \& Gomes, W. B. (2004). Estou me formando... e agora?: reflexões e perspectivas de jovens formandos universitários. Revista Brasileira de Orientação Profissional, 5(1), 47-62. Recuperado em março 4, 2014, de http://pepsic.bvsalud.org/scielo.php? script=sci_arttext\&pid=S1679-33902004000100 005\&lng=pt\&nrm=iso\&tlng=pt

Teixeira, M. A. P., \& Gomes, W. B. (2005). Decisão de carreira entre estudantes em fim de curso universitário. Psicologia: Teoria e Pesquisa, 21(3), 327-334.

Viriguine, N. R., Krawulski, E., Tavares, G., \& Soares, D. H. P. (2010). Da formação superior ao mercado de trabalho: percepções de alunos sobre a disciplina orientação e planejamento de carreira em uma universidade federal. Revista Electrónica de Investigación y Docencia, 4, 79-96.

Yamamoto, O. H., Souza, J. A. J., Silva, N., \& Zanelli, J. C. (2010). A formação básica, pós-graduada e complementar do psicólogo no Brasil. In A. V. B. Bastos \& S. M. G. Gondim (Orgs.), O trabalho do psicólogo no Brasil (pp.45-65). Porto Alegre: Artmed.

Zanelli, J. C., \& Bastos, A. V. B. (2004). Inserção profissional do psicólogo em organizações e no trabalho. In J. C. Zanelli, J. E. Borges-Andrade, \& A. V. B. Bastos (Orgs.), Psicologia, organizações e trabalho no Brasil (pp.466491). Porto Alegre: Artmed.

Zanon, C., Bastianello, M. R., Pacico, J. C., \& Hutz, C. S. (2013). Relationships between positive and negative affect and the five factors of personality in a Brazilian sample. Paidéia, 23(56), 285-292. http://dx.doi.org/ 10.1590/1982-43272356201302

Recebido: setembro 12, 2014

Versão final: janeiro 26, 2015

Aprovado: abril 7, 2015 